\title{
Numerical simulation of aqueous humor flow: from healthy to pathologic situations
}

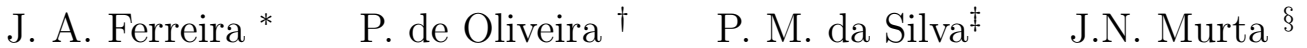

\begin{abstract}
A mathematical model which simulates drug delivery through the cornea, from a therapeutic lens to the anterior chamber of the eye, is proposed. The model consists of three coupled systems of partial differential equations linked by interface conditions: drug diffusion in the therapeutic lens; diffusion and metabolic consumption in the cornea; diffusion, convection and metabolic consumption in the anterior chamber of the eye. The dependence of intraocular pressure on the obstruction of the trabecular mesh and the production rate of aqueous humor by the ciliary body is modeled. The therapeutic effects of drugs that act on the trabecular mesh or on the ciliary body are analysed. Comparisons between topical administration and drug delivery from a therapeutic lens are included.
\end{abstract}

\section{Introduction}

The anterior segment the eye is filled with a clear fluid called aqueous humor (AH) that is composed predominantly of water. It has two main roles: to deliver oxygen and nutrition to tissues within the eye; and to maintain a correct balance and regulation of intraocular pressure (IOP), which is important in early ocular development as well as maintaining integrity throughout life. The fluid is continuously produced in the ciliary body. It leaves the eye through the trabecular meshwork (TM), sievelike structure, and converts into a tube called the Schlemm's canal, located in angle of the anterior chamber. The aqueous humor flows through this drainage structure and mixes with venous blood (Figure 1). Consequently, the two main structures related to the dynamics of $\mathrm{AH}$ are the ciliary body - the site where $\mathrm{AH}$ is produced - and the limbal region, which includes the trabecular meshwork - that regulates its outflow $([1],[2])$. Clinical research points out that "the combined resistance of the TM and the Schlemm's canal are the primary source of resistance in outflow". This pathway accounts for about $70 \%-90 \%$ of aqueous outflow.

\footnotetext{
${ }^{*}$ CMUC-Department of Mathematics, University of Coimbra. E-mail:ferreira@mat.uc.pt.

${ }^{\dagger}$ CMUC-Department of Mathematics, University of Coimbra. E-mail:poliveir@mat.uc.pt.

${ }^{\ddagger}$ Instituto Politécnico de Coimbra, ISEC, DFM, Rua Pedro Nunes, 3030-199 Coimbra, Portugal. CMUC. E-mail:pascals@isec.pt.

${ }^{\S}$ Faculty of Medicine, University of Coimbra.
} 


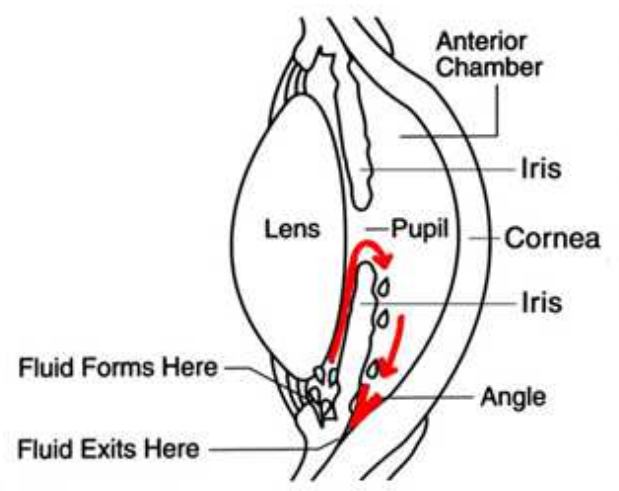

Figure 1: Anatomy of the eye (http://www.theeyecenter.com/educational/005.htm).

Intraocular pressure (IOP) is the result of a complex interplay of the components of aqueous humor dynamics. When an obstruction of the TM or the Schlemm's canal occur an increase of the IOP is then observed. Several studies have shown evidence that IOP can be

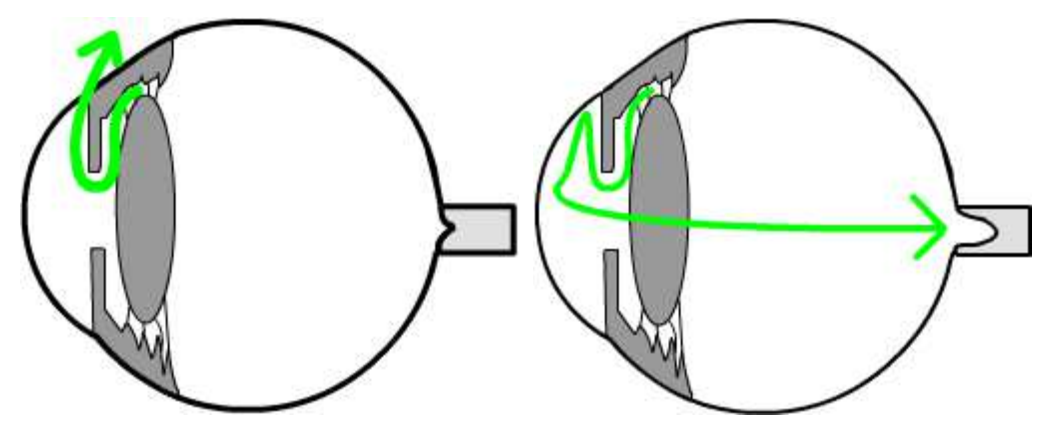

Figure 2: Increase of IOP and damage of the optic nerve - rectangle shaped structure on the right side of the schemes (http://www.thecontactlenspractice.co.uk/page/glaucoma/).

controlled by decreasing the production of $\mathrm{AH}$ and/or decreasing the grade of obstruction of such structures $([3],[4])$.

Glaucoma is a progressive optic nerve neuropathy where the direct mechanical effect of an increase of IOP is the most common risk factor for its progression (Figure 2). It can result in vision loss and it is one of the leading causes of blindness. Glaucoma is the $3^{\text {rd }}$ most common cause of blindness worldwide and the $2^{\text {nd }}$ most common cause of blindness in the US and Europe and the disease is expected to affect more and more people as elderly population grows. With early treatment it can be controlled and therefore its diagnosis is crucial.

Open angle glaucoma is characterized by an obstruction of the drainage structures and an increase in the IOP is a major risk factor, lowering this pressure is the principal option of treatment in this clinical situation. In closed angle glaucoma the iris moves forward from its normal position and reduces drastically or completely eliminates the camerular angle (space 
between the iris and the cornea). It causes a total obstruction of AH outflow and consequently a sudden increase of IOP. In this study we only consider open angle glaucoma and its treatment with beta-blockers (which decrease the $\mathrm{AH}$ production rate) and prostaglandin derivatives (that increase the uveal-scleral outflow).

As the behaviour of IOP is mainly related with the dynamics of $\mathrm{AH}$ in the anterior chamber of the eye, computational simulations in healthy and pathologic conditions can be a very useful tool for clinicians and pharmaceuticals. Several studies have addressed recently the dynamics of AH ([5], [6], [7], [8], [9]). However at the best of our knowledge the interaction of drug flow with the dynamics of $\mathrm{AH}$ and the effects on IOP decrease of different families of drugs have not been modelled and simulated so far. The effects on IOP of the production rate of $\mathrm{AH}$ and the obstruction of TM as well as the coupling of $\mathrm{AH}$ flow with drug delivery mechanisms and transport mechanisms through the cornea have not been described so far in the literature. The findings of these studies clearly the mechanisms of IOP variations and they can give indications of how to tailor a treatment in order to fit specific patient's needs.

To simulate a treatment the type of drug and the release mechanism must be specified. Several families of drugs are used to lower the intraocular pressure (IOP) and consequently to prevent optic nerve damage. We mention without being exhaustive: beta-blockers that reduce $\mathrm{AH}$ production; prostaglandin derivatives which increase the outflow of $\mathrm{AH}$; alpha agonists and combined medications that decrease the production of $\mathrm{AH}$ and simultaneously increase its outflow ([10], [11], [12], [3], [13]). In the present paper we simulate the therapeutic effect of beta-blockers and prostaglandin derivatives. As far as drug release mechanisms are concerned two procedures are clinically used: ophthalmic drops and therapeutic lens. Ophthalmic drops are by far the most used route of drug delivery to the eye. However it is estimated that when a drop is instilled into the eye it is diluted by the lachrymal secretion and $95 \%$ is cleared by the tear fluid. To improve the efficiency of drug delivery avoiding drug loss and also deleterious side effects many researchers have proposed the use of therapeutic contact lenses as a vehicle to deliver ophthalmic drugs ([4], [14], [15], [16], [17], [18]). The method is particularly important in the case of severe diseases of the eye, as for example glaucoma, accompanying population ageing for which long periods of drug delivery are needed ([21]). The main advantage of this route is the possibility of controlling drug delivery by means of the use of polymeric lens designed to achieve pre-defined performances with a high degree of comfort and biocompatibility.

In a previous paper ([16]) the authors modeled drug delivery from a therapeutic lens, through the cornea. A one dimensional space model was used in the therapeutic lens and in the cornea. The behaviour of the AH in the anterior chamber was modeled by an ordinary differential equation, and consequently its anatomical characteristics and the patterns of $\mathrm{AH}$ flow were not taken into account. However to describe in vivo dynamics of $\mathrm{AH}$ and the effect of specific treatments, the flow patterns and the geometry of the anterior chamber in 
healthy and pathologic situations must be analysed. In this context clinical and laboratorial results are not available in many situations and for this reason numerical simulations are an important tool to suggest new routes of research in the treatment of glaucoma. All the simulations included in this paper have been obtained with the finite element solver COMSOL Multiphysics version 4.2 .

In this paper we begin by addressing in Section 2 the dynamics of $\mathrm{AH}$, described by Navier stokes and Darcy's law, and its dependence on two main control parameters: the $\mathrm{AH}$ inflow and outflow rates. In Section 3 we establish a set of coupled system's of partial differential equations which simulate the treatment of high IOP. Two drug delivery procedures - topical administration and drug delivery from a therapeutic lens - are compared. The numerical results obtained showed that whereas with topical drops there is an initial burst in the concentration, with the therapeutic lenses the delivery is sustained, lasting for longer periods of time. As a consequence the treatments in Section 3.2 will be simulated considering that the drug is delivered from a therapeutic lens. In Subsection 3.2.1 the effect of drugs that decrease the inflow rate (beta-blockers) is modeled. In Subsection 3.2.2 the decrease in IOP caused by drugs that increase the outflow rate is simulated. Finally in Section 4 we compare our mathematical findings with clinical indications.

\section{Aqueous Humor flow and intraocular pressure}

\subsection{Geometry}

The eye is divided into three chambers: the vitreous chamber filled with a gel like material called the vitreous humor and the anterior and posterior chambers, filled with AH.

In Figure 4 we represent a 2D geometry resulting from an horizontal section, $\Omega_{1}$, of the anterior chamber of the eye. The geometrical model used in this paper is based on physiological dimensions of the human eye ([1]). The section of the cornea is represented by $\Omega_{4}$ and the section of TM is represented by $\Omega_{2}$ and $\Omega_{3}$. We note that as TM, represented in Figure 3, in an annular structure (in the basis of the cornea) its section is a disconnected set $\Omega_{2} \cup \Omega_{3}$. The external boundaries of the domains as well as the interfaces are signaled in the Figure 4. We describe in what follows such boundaries and interfaces.

The $\mathrm{AH}$ is assumed to be a linear viscous liquid with properties close to those of water. The cornea is modeled as a rigid shell with a thickness of $0.5 \mathrm{~mm}$ along the central axis. It is a vascular and transparent tissue with thermal properties close to that of water and which is kept at a constant temperature of $310.15 K$ in the present model.

The depth of the anterior chamber of the human eye is approximately $3 \mathrm{~mm}$ and the diameter in the plane of the iris root is $11-12 \mathrm{~mm}$ [1]. The boundaries of the anterior chamber $\Omega_{1}$, are modeled using a Bezier curve with $3 \mathrm{~mm}$ of thickness along the central axis and $12 \mathrm{~mm}$ of diameter. The anterior chamber is surrounded by the inner surface of the 


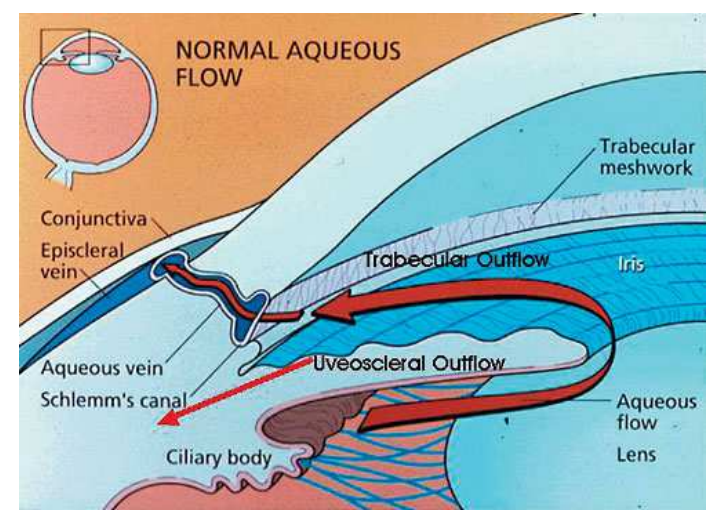

Figure 3: Anatomy of the eye: trabecular mesh (http : //www2.cfpc.ca/cfp/2005/Sep/vol51 - sep - cme $-3_{f}$ r.asp).

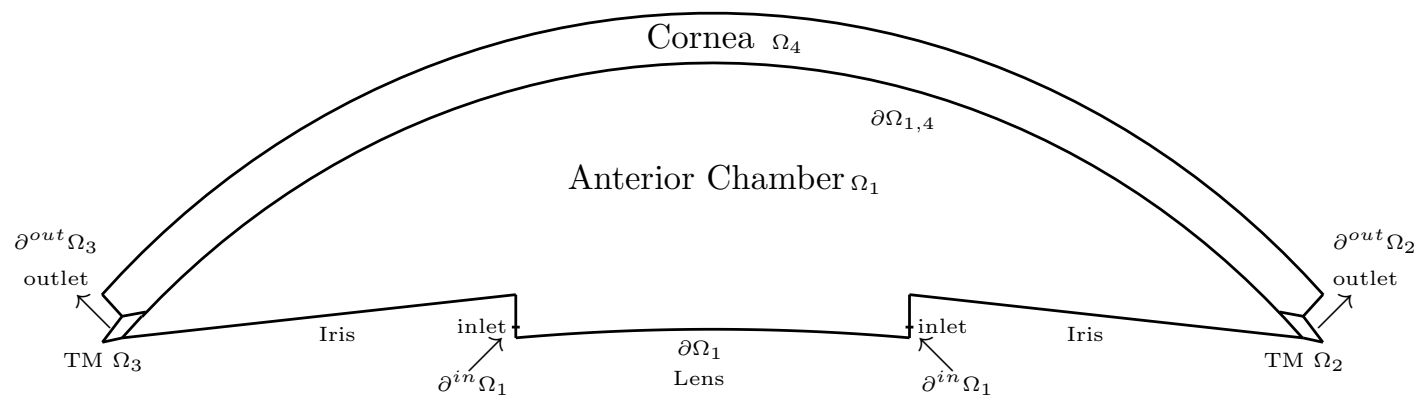

Figure 4: Geometry of the model: anterior chamber $\left(\Omega_{1}\right)$, trabecular mesh $\left(\Omega_{2}\right.$ and $\left.\Omega_{3}\right)$ and cornea $\Omega_{4}$.

cornea, $\partial \Omega_{1,4}$, and by the inner surfaces of the TM, represented by $\partial \Omega_{1,2}$ and $\partial \Omega_{1,3}$ (Figure 8). On the posterior side, the anterior surface of the iris and the pupillary portion of the anterior lens surface $\left(\partial \Omega_{1}\right)$ bound the anterior chamber.

The AH flow enters into the anterior chamber from the posterior chamber where it is secreted by the ciliary body at a constant volume flow rate of $2.5 \mu \mathrm{L} / \mathrm{min}$. More precisely the AH flows through the small gap between the iris and lens $\left(\partial^{i n} \Omega_{1}\right)$ which is estimated to be a few microns wide $(10-25 \mu \mathrm{m})$. The AH leaves the TM through the outlet boundary $\partial^{\text {out }} \Omega_{2}$, $\partial^{\text {out }} \Omega_{3}$ and it is collected into the Schlemm's canal.

We present in Annex A the units of all the variables and parameters used in the model.

\subsection{Mathematical model of aqueous humor flow}

To simulate the dynamics of $\mathrm{AH}$ in the anterior chamber we take into account the porous structure of the TM. We assume, as generally accepted in fluid mechanics literature, that 
all significant aspects of an incompressible or compressible flow can be accurately studied by Navier-Stokes equation. Aqueous humor is mainly composed by water $(99 \%)$ so we describe its kinetics - as for example do the authors in [6], [7], [8], [9],[19] and [20], - by the incompressible Navier Stokes equation in $\Omega_{1}$ coupled with Darcy's law in $\Omega_{2}$ and $\Omega_{3}$, as follows:

$$
\begin{aligned}
& \left\{\begin{array}{c}
\rho \frac{\partial \mathbf{v}}{\partial t}-\nabla \cdot \mu\left(\nabla \mathbf{v}+(\nabla \mathbf{v})^{T}\right)+\rho(\mathbf{v} \cdot \nabla) \mathbf{v}+\nabla p=0, \text { in } \Omega_{1}, t>0 \\
\nabla \cdot \mathbf{v}=0, \text { in } \Omega_{1}, t>0
\end{array},\right. \\
& \left\{\begin{array}{c}
\mathbf{v}=-\frac{K}{\mu} \nabla p, \text { in } \Omega_{2}, \Omega_{3}, t>0 \\
\nabla \cdot \mathbf{v}=0, \text { in } \Omega_{2}, \Omega_{3}, t>0
\end{array} .\right.
\end{aligned}
$$

In the Navier-Stokes equation (1) $\mathbf{v}$ represents the velocity of AH, $\rho$ its density, $\mu$ the viscosity and $p$ the intraocular pressure. In Darcy's law (2) the permeability, $K$, is considered as an increasing function of the porosity of the TM,

$$
K=\frac{D_{p}^{2} \epsilon^{3}}{150(1-\epsilon)^{2}}
$$

where $D_{p}$ is the pore size and $\epsilon$ is the porosity ([5]).

System (1-2) is coupled with boundary conditions of the following type:

- Inflow boundary condition for the velocity, $\mathbf{v}=v_{0}$. We note that the normal value $v_{0}$ of the velocity at the inlet of the anterior chamber is around $1.2 \times 10^{-3} \mathrm{~m} / \mathrm{s}$ and it is related with a normal production rate of the $\mathrm{AH}$ in the ciliary body;

- Outflow boundary conditions for the pressure, $p=p_{0}$, where $p_{0}$ is the pressure observed in the Schlemm's canal. It has the same value as the pressure in the blood. In our simulations $p_{0}=1200 P a$.

- Interface boundary continuity conditions for the pressure, in the two interface boundaries $\partial \Omega_{1,2}$ and $\partial \Omega_{1,3}$;

- Wall conditions, $\mathbf{v}=0$, in the boundary of $\Omega_{1}$ excepting $\partial^{i n} \Omega_{1} \cup \partial \Omega_{1,2} \cup \partial \Omega_{1,3}$ (Figure $8)$.

Equations (1-2) are completed with initial conditions represented by

$$
\left\{\begin{array}{c}
\mathbf{v}=0, \text { in } \Omega_{1}, t=0 \\
p=1950, \text { in } \Omega_{1}, t=0
\end{array} .\right.
$$

Using normal values for the pressure in the $\mathrm{AC}(1950 \mathrm{~Pa})$ and in the Schlemm's canal $(1200 \mathrm{~Pa})$ an interpolating function is defined to prescribe the initially pressure in the TM. 
AH undergoes a constant turnover: it is produced by the ciliary body and it is drained through the TM. In the ophtalmology literature it is referred that small variations occurring in the production rate of $\mathrm{AH}$ or in its drainage can produce large variations in the IOP and consequently damage of the optic nerve. IOP can be kept under control by either reducing the production of $\mathrm{AH}$ or by increasing its outflow. Thus understanding these two phenomena is essential for the management of glaucoma.

In section 2.3 we study numerically the influence of $\mathrm{AH}$ production rate on IOP. The influence of drainage on the variation of IOP is analysed in Section 2.4.

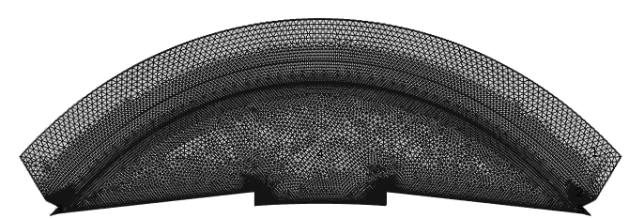

Figure 5: Mesh of the total domain: therapeutical lens, cornea, anterior chamber and trabecular mesh.

In the numerical simulations of this section and Section 3 the finite element solver COMSOL Multiphysics, version 4.2, is used. Spatial discretization in a non regular mesh is based on P2 finite elements (Figure 5). We note that the mesh, with 19070 elements, is highly refined in the inflow region, outflow region and interfaces between different layers of the domain. Integration in time uses variable Backwards Difference Formulae with variable time step and order between 1 and 2. The non linear systems resulting from the discretization are solved with Multifrontal Massively Paralel Sparse direct solver (MUMPS).

\subsection{The influence of aqueous humor production rate on IOP}

As mentioned before current therapies for lowering IOP include increasing the AH outflow and/or decreasing the $\mathrm{AH}$ production. We analyse in this subsection the influence on IOP of $\mathrm{AH}$ production rate. To simulate such influence we compute the steady state pressure in problem (1-2) for different inlet velocities. The velocity of $\mathrm{AH}$ in the steady state is represented in Figure 6, where the streamlines show the path of the flow. We study two different situations: a healthy eye (with the permeability given by $K=7 \times 10^{-15}$ and the porosity by $\epsilon=0.21604$ ) and a pathologic situation (with $K=2.3 \times 10^{-15}$ and $\epsilon=0.15653$ ). In Table I we present the values obtained for the IOP, the steady state intraocular pressure, 


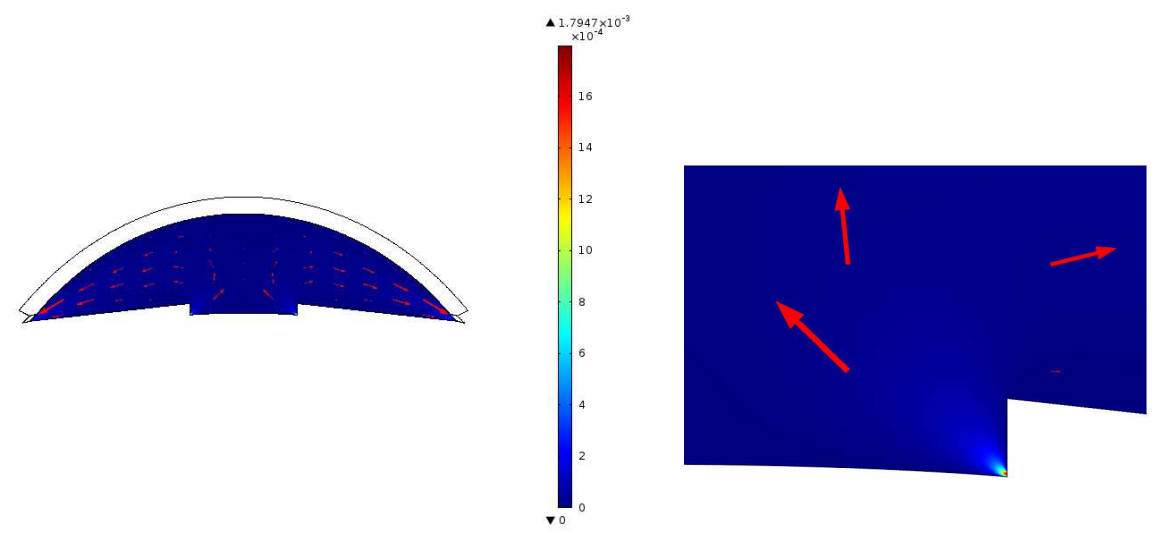

Figure 6: Velocity of the aqueous humor in the anterior chamber, with a normal interior pressure $\left(v_{0}=1.2 \times 10^{-3} \mathrm{~m} / \mathrm{s}, P=7 \times 10^{-15} \mathrm{~Pa}\right)$.

when different values of the inflow velocity are considered with $\mu$ and $\rho$ the corresponding water viscosity and density at the temperature of $310.15 \mathrm{~K}$. We note that $v_{0}=1.2 \times 10^{-3} \mathrm{~m} / \mathrm{s}$, which is the normal value of the velocity.

Analyzing Table I we conclude that the variation of the production rate of AH produces a more significant effect in a pathologic situation than in a healthy eye.

Table I - IOP in a healthy and pathologic eye, for different values of inflow velocity.

\begin{tabular}{||c|c||c||}
\hline \hline Velocity $(\mathrm{m} / \mathrm{s})$ & Pressure in a pathologic eye $(P a)$ & Pressure in a healthy eye $(P a)$ \\
\hline$\frac{v_{0}}{2}$ & 2548 & 1585 \\
$v_{0}$ & 3896 & 1970 \\
$1.25 v_{0}$ & 4570 & 2163 \\
$1.5 v_{0}$ & 5244 & 2356 \\
$1.75 v_{0}$ & 5917 & 2548 \\
$2 v_{0}$ & 6591 & 2741 \\
\hline \hline
\end{tabular}

In Figure 7 we observe that a healthy eye is not as sensible as a pathologic eye to variations of the $\mathrm{AH}$ production rate and for this reason the $\mathrm{AH}$ production rate can be used to control the decrease of IOP.

\subsection{The influence of aqueous humor drainage on intraocular pressure}

The AH leaves the anterior chamber, to enter in the blood system, through the TM. The increased resistance of the TM is represented in our model by the obstruction of the TM that occurs when the porosity and permeability of the structure decrease.

The AH leaves the anterior chamber through the TM to the Schlemm's canal by the two outlet boundaries $\partial^{\text {out }} \Omega_{2}$ and $\partial^{\text {out }} \Omega_{3}$, represented in Figure 4 and zoomed in Figure 8. We will analyze the implications of such obstruction in the buildup of IOP. As expected, a decrease 


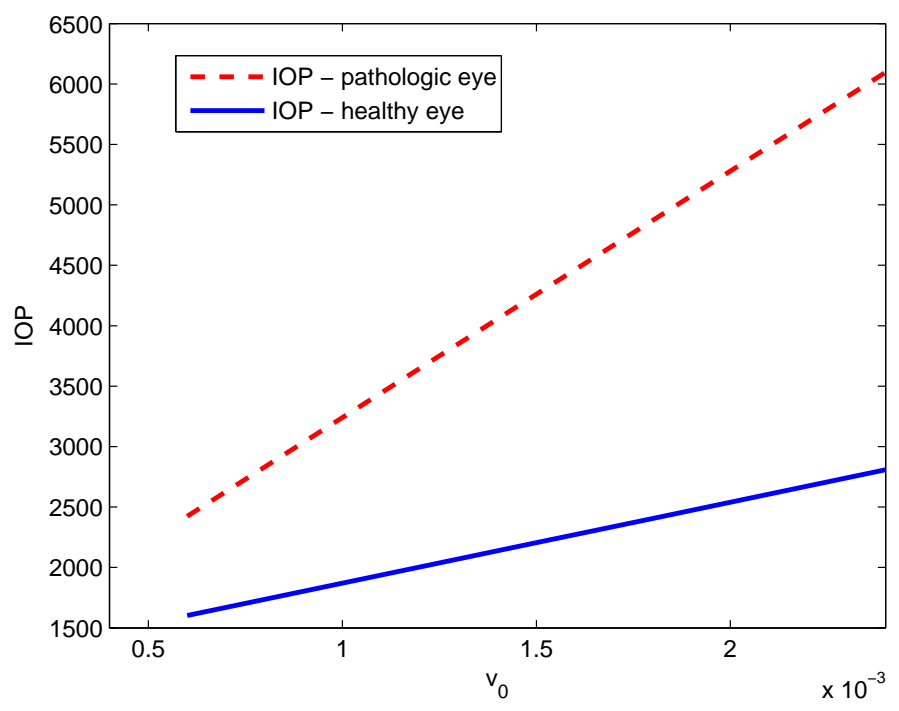

Figure 7: IOP in the anterior chamber as a function of of the AH production rate.

in the porosity of the TM implies an increase of the pressure in the anterior chamber. To establish a quantitative relationship between IOP and the grade of obstruction of the TM, we exhibit in Table II the "route" from a healthy eye to the rise of an open-angle glaucoma. In open angle glaucoma the outflow boundaries present an high flow resistance.

The values in Table II have been obtained by solving equations (1-2), with different values of the porosity $\epsilon$.

From a medical point of view a value of IOP higher than $25 \mathrm{mmHg}$ (approximately $3333 \mathrm{~Pa}$ ) is a critical value and the patient can be suspected of glaucoma mainly if typical visual field defects and abnormal optic nerve on ophthalmoscopy are observed. Normal pressure in the anterior chamber is quite close to $1800-2200 \mathrm{~Pa}$.

Table II - IOP for different grades of obstruction of resistance of the TM.

\begin{tabular}{||c|c|c||}
\hline \hline Porosity $(\epsilon)$ & Permeability $\left(\mathrm{m}^{2}\right)$ of TM & Pressure in anterior chamber $(P a)$ \\
\hline 0.4 & $7.59 \times 10^{-14}$ & 1271 \\
0.3 & $2.35 \times 10^{-14}$ & 1429 \\
0.25 & $1.19 \times 10^{-14}$ & 1655 \\
0.225 & $8.09 \times 10^{-15}$ & 1867 \\
0.2 & $5.33 \times 10^{-15}$ & 2211 \\
0.175 & $3.36 \times 10^{-15}$ & 2805 \\
0.15 & $1.99 \times 10^{-15}$ & 3905 \\
0.125 & $1.09 \times 10^{-15}$ & 6154 \\
0.1 & $5.27 \times 10^{-16}$ & 11437 \\
\hline \hline
\end{tabular}



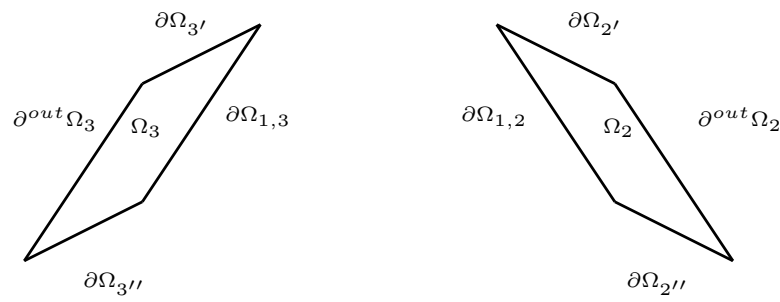

Figure 8: Zoom of the Trabecular mesh, $\Omega_{2} \cup \Omega_{3}$, represented in Figure 4 and its boundaries: - Interface boundaries with $\partial \Omega_{1,3}$ (left) and $\partial \Omega_{1,2}$ (right), Outlet boundaries $\partial^{\text {out }} \Omega_{3}$ (left) and $\partial^{\text {out }} \Omega_{2}$ (right) .

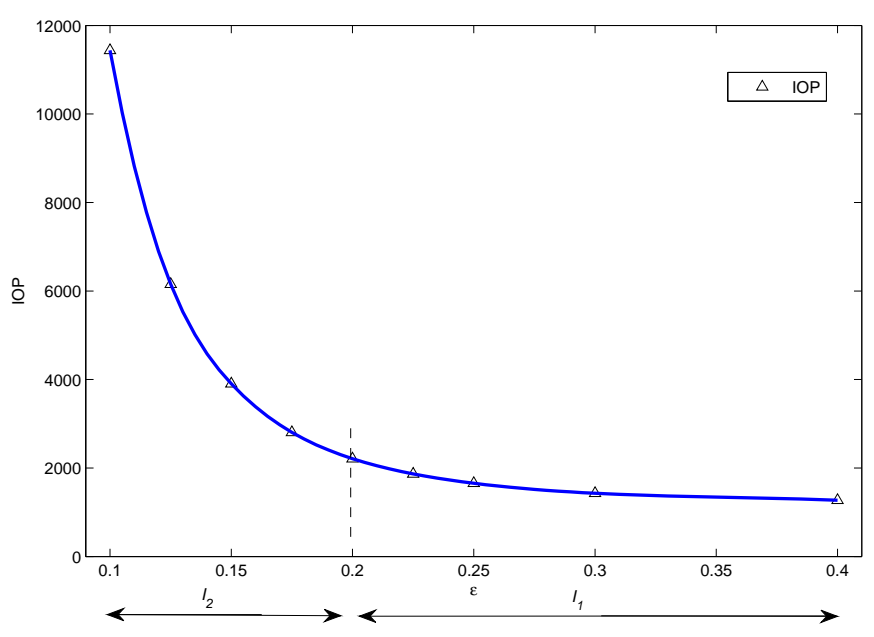

Figure 9: Dependence of IOP on the porosity $\epsilon$.

We note that the IOP suffers a slight increase when the permeability $K$ is in the interval $I_{1}=\left[7.59 \times 10^{-14}, 5.33 \times 10^{-15}\right]$. The IOP exhibits a steep gradient when $K$ is in the interval $I_{2}=\left[3.36 \times 10^{-15}, 5.27 \times 10^{-16}\right]$. These findings are in agreement with the fact that for clinicians the diagnosis of glaucoma does not simply rely on the presence of high IOP. In fact as can be observed in Table II and Figure 9 a progressive resistance is occurring in interval $I_{1}$ but no significant increase of IOP is observed. This remark also explains why low IOP is not enough to preclude the presence of glaucoma.

In Figure 10 we represent the steady state of pressure in the TM for a eye with a normal value of porosity, $\epsilon=0.225$. We can observe in the zoom of the TM that near the interface with the anterior chamber the pressure is approximately $1867 \mathrm{~Pa}$ which is the value of IOP in the anterior chamber (Table II). 

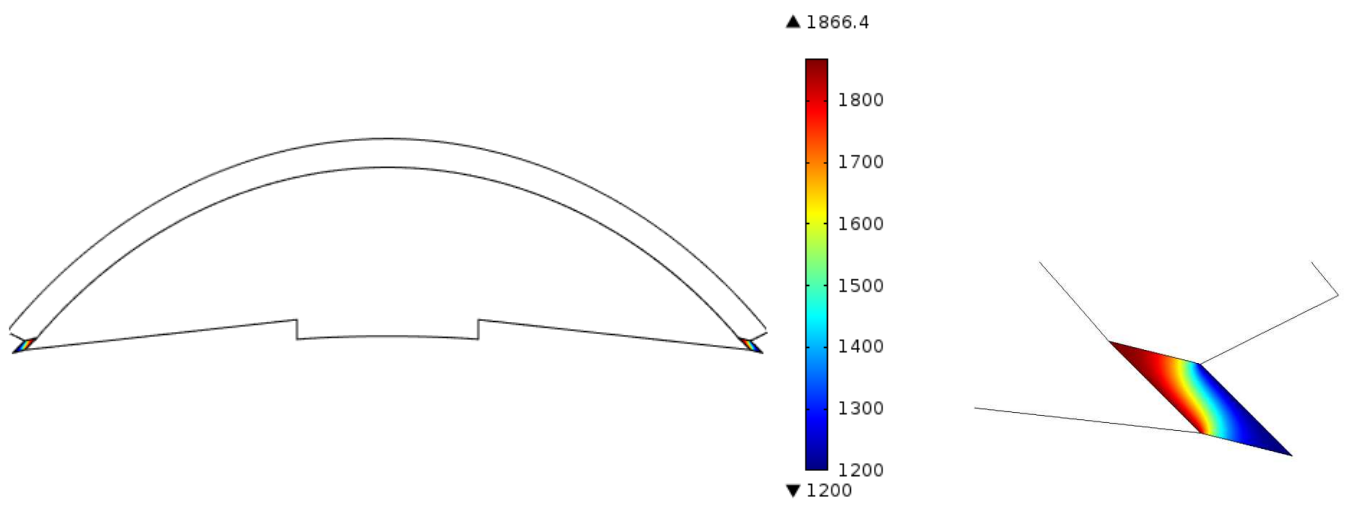

Figure 10: IOP in the trabecular mesh in a normal eye - right; zoom of IOP in the TM - left.

\section{Treatment simulations: Drugs with therapeutic effect in glaucoma}

\subsection{Drug distribution in the anterior chamber: from topical administration to therapeutic lens}

The aim of this subsection is to compare the evolution of drug concentration in the anterior chamber, when it is delivered from a therapeutic lens or when drops are administered. We begin by assuming that the drug has no therapeutical effect on glaucoma, that is the rates of production and drainage of $\mathrm{AH}$ are kept constant. From a mathematical point of view this means that the boundary condition $\mathbf{v}=v_{0}$ in $\partial^{i n} \Omega_{1}$ is not change in time and also the porosity, $\epsilon$, is constant. For this reason and to keep the model as simple as possible the TM is not considered in the geometry used in this subsection (Figure 11).

The drug delivery from a therapeutic lens is modeled by three coupled systems of partial differential equations, linked by interface conditions, that simulate the evolution of drug concentration in the therapeutic lens, the cornea and the anterior chamber.

We begin by considering the system that represents the drug evolution in a therapeutic lens. The platform presented in [17] and [4], where the drug is dispersed in a polymeric lens and also entrapped inside particles dispersed in the lens, was lately modified in [15], [16] and [18]. The numerical simulations presented in this paper use the lens model in [15], [16]. Any other type of therapeutic lens can be considered as for example the recent prototype by Ciolino and co-authors presented in [14].

The evolution of drug concentration in the lens is described by the system of PDE's

$$
\left\{\begin{array}{c}
\frac{\partial C}{\partial t}=D_{l} \nabla^{2} C-\lambda\left(C-C^{b}\right), \text { in } \Omega_{5}, t>0 \\
\frac{\partial C^{b}}{\partial t}=D_{b} \nabla^{2} C^{b}+\lambda\left(C-C^{b}\right), \text { in } \Omega_{5}, t>0
\end{array},\right.
$$




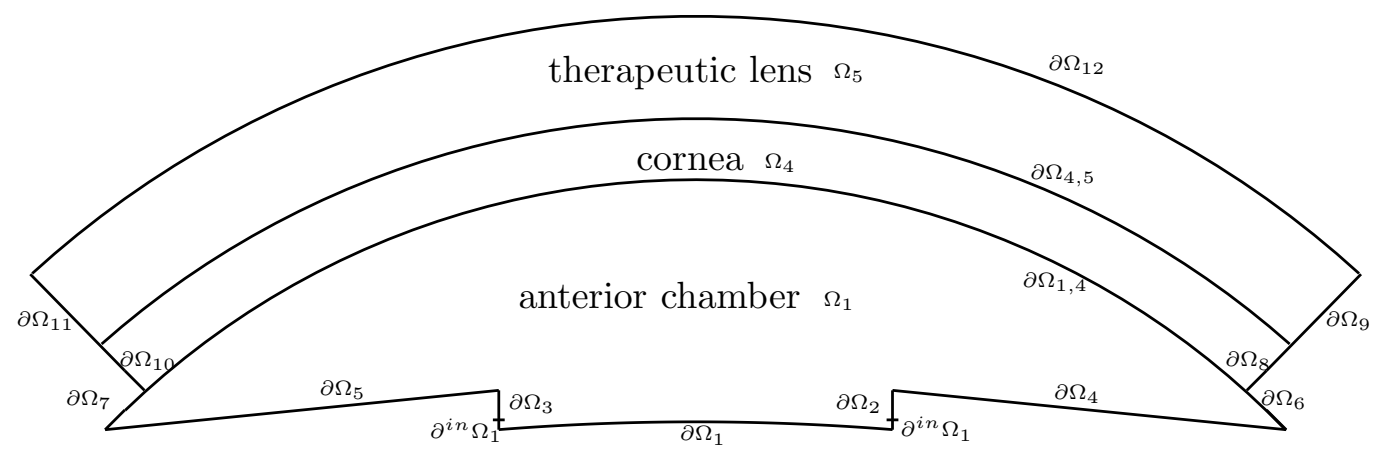

Figure 11: Geometry of the model: therapeutic lens $\left(\Omega_{5}\right)$, cornea $\left(\Omega_{4}\right)$ and anterior chamber $\Omega_{1}$.

where $C$ and $C^{b}$ represent respectively the free drug concentration and the concentration inside the particles, $D_{l}$ and $D_{b}$ stand for the diffusion coefficient of the drug in the polymeric matrix and in the particles and $\lambda$ is the transference coefficient of the drug between the particles and the polymeric lens.

The kinetics in the cornea, driven by diffusion and metabolic consumption, is described by the diffusion-reaction equation

$$
\frac{\partial C_{c}}{\partial t}=D_{c} \nabla^{2} C_{c}-K_{c} C_{c}, \text { in } \Omega_{4}, t>0,
$$

where $C_{c}$ represents the drug concentration in the cornea, $D_{c}$ stands for the diffusion coefficient in the cornea and $K_{c}$ represents a coefficient that takes into account the metabolic consumption of the drug. As previously mentioned we did not consider the cornea divided in epithelium, stroma and endothelium. Also the corneal thickness was not taken into account. In our model $D_{c}$ and $K_{c}$ represent the mean value of the diffusion coefficients and the metabolic consumption in these tissues, respectively. The same assumptions are considered in the mathematical models of topical administration introduced in [22] and considered later in [23].

To simulate the evolution of drug concentration in the anterior chamber we take into account the phenomena of diffusion, convection and metabolic consumption. The whole kinetics of the drug is then described by

$$
\frac{\partial C_{a}}{\partial t}=D_{a} \nabla^{2} C_{a}-\mathbf{v} \cdot \nabla C_{a}-\frac{C_{l a}}{V_{a}} C_{a}, \text { in } \Omega_{1}, t>0,
$$


where $C_{a}$ represents the drug concentration in the anterior chamber, $D_{a}$ stands for the diffusion coefficient, $\mathrm{Cla}$ is the clearance of drug in the anterior chamber and $V_{a}$ represents the distribution volume of solute in the anterior chamber. In the last equation $\mathbf{v}$ represents the velocity of the $\mathrm{AH}$, that is given by equation (1).

Equations (4-6) are completed with initial conditions represented by

$$
\left\{\begin{array}{c}
C=C_{0}, \text { in } \Omega_{5}, t=0 \\
C^{b}=C_{0}^{b}, \text { in } \Omega_{5}, t=0 \\
C_{c}=0, \text { in } \Omega_{4}, t=0 \\
C_{a}=0, \text { in } \Omega_{1}, t=0
\end{array},\right.
$$

and the following boundary conditions

$$
\left\{\begin{array}{c}
D_{l} \nabla C \cdot \eta=0, \text { in } \partial \Omega_{i},{ }_{i=9,11,12}, t>0 \\
D_{b} \nabla C^{b} \cdot \eta=0, \text { in } \partial \Omega_{i}, i=9,11,12 \text { and in } \partial \Omega_{4,5}, t>0 \\
D \nabla C \cdot \eta=D_{c} \nabla C_{c} \cdot \eta, \text { in } \partial \Omega_{4,5}, t>0 \\
D_{c} \nabla C_{c} \cdot \eta=0, \text { in } \partial \Omega_{i},{ }_{i=8,10}, t>0 \\
D_{c} \nabla C_{c} \cdot \eta=A_{c}\left(C_{c}-C a\right), \text { in } \partial \Omega_{1,4}, t>0 \\
D_{a} \nabla C_{a} \cdot \eta=0, \text { in } \partial \Omega_{i},{ }_{i=1,2,3,4,5}, t>0
\end{array} .\right.
$$

The first two conditions in (7) indicate the initial concentrations in the lens (in the polymeric matrix and in the dispersed particles). The two other conditions state that at $t=0$ there is no drug in the eye (cornea and anterior chamber). In (8) we have three type of conditions: impermeable walls, flux continuity and Robin types conditions. The definition of the boundary conditions in $\partial^{i n} \Omega_{1}$, where the $\mathrm{AH}$ enters in the anterior chamber, and in $\partial \Omega_{i},{ }_{i=6,7}$, where it leaves the anterior chamber, are the following:

$$
\left\{\begin{array}{c}
v=v_{0}, \text { in } \partial^{i n} \Omega_{1}, t>0 \\
p=p_{0}, \text { in } \partial \Omega_{i},{ }_{i=6,7}, t>0
\end{array} .\right.
$$

We solve problem (1,4-6) coupled with initial condition (7) and boundary conditions (8)(9).

The outflow boundaries $\partial \Omega_{6}$ and $\partial \Omega_{7}$ represent the interface between the anterior chamber and the TM (Figure 11). In Section 2.2 the grade of obstruction of the TM is simulated considering different values for the porosity $\epsilon$ (Table II). In this section, as already mentioned, we do not include the geometry of the TM. Its obstruction corresponding to a pathologic situation, is represented by assuming that the outflow only occurs through a part of such interfaces which we call the viable length. Varying the viable length of $\partial \Omega_{6}$ and $\partial \Omega_{7}$ we compute the corresponding steady pressure $p$. A viable length leading to $p=3664 P a$ has been selected. We note that this value of IOP is much higher than the value of a normal pressure for a healthy eye $(2000 P a)$. 
To simulate the evolution of drug concentration when optical drops are instilled in the eye we replace (4) by an equation that describes the evolution of drug in the tear film

$$
\frac{\partial C_{T}}{\partial t}=D_{a} \nabla^{2} C_{T}-\frac{S}{V_{L}+V_{i} e^{-k_{d} t}} C_{T}, \text { in } \Omega_{1}, t>0 .
$$

In (10) $C_{T}$ denotes the drug concentration in the tear film, $S$ represents the (fixed) lacrimal secretion rate, $k_{d}$ denotes the drainage constant and $V_{L}$ and $V_{i}$ represent respectively the normal lacrimal volume and the initial tear volume after an instillation of drug. We remark that $\Omega_{1}$ in this case represents the tear film.

To compare the two procedures of drug administration, we exhibit in Figure 12 plots of drug concentration at $t=20$ minutes when drops are instilled (a) or therapeutic lenses are used (b).

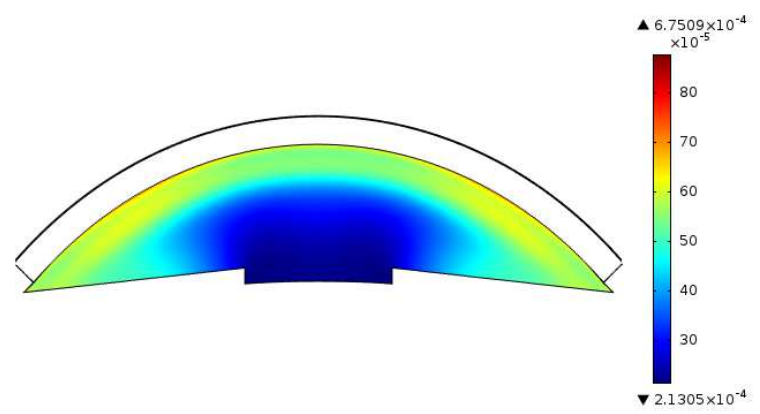

(a)

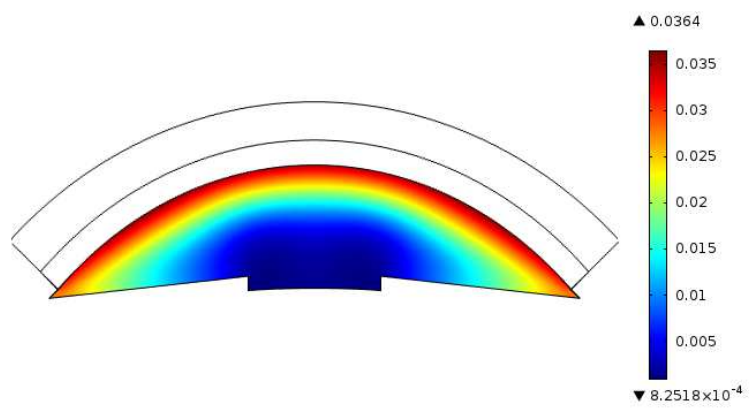

(b)

Figure 12: Drug concentration in the anterior chamber at $t=20 \mathrm{~min}$, when we use a drop (a) and a therapeutic lens (b), in a pathologic situation.

The numerical results have been obtained with $C_{0}=1.147, C_{0}^{b}=0.209$, that represent respectively the concentrations in the lens and the particles; $D_{l}=3.333 \times 10^{-11}, D_{c}=$ $5.74 \times 10^{-9}, D_{a}=5 \times 10^{-11}$, that represent the diffusion coefficients $\left(\mathrm{m}^{2} / \mathrm{seg}\right)$ of the drug in the lens, cornea and anterior chamber, respectively. The following values for the parameters:

$$
\lambda=2 \times 10^{-4}, K_{c}=1.0713 \times 10^{-5}, A_{c}=0.1, C l a=30, V_{a}=1000,
$$

have been considered. The numerical simulations representing the topical administration have been obtained with

$$
k d=1.45 \mathrm{~min}^{-1}, C_{T}^{0}=0.5 \times 10^{-3} \mathrm{~mol} / \mathrm{m}^{3}, V L=7 \mu l, V_{i}=10 \mu l, S=1.2 \mu l / \mathrm{min} .
$$

We remark that the lowest drug concentration observed in the anterior chamber when a therapeutic lens is used Figure 12 (b), $8.25 \times 10^{-4} \mathrm{~mol} / \mathrm{m}^{3}$, is larger than the highest values 
of the drug concentration when a drop is administered to the eye Figure 12 (a), (6.75 $\times$ $\left.10^{-4} \mathrm{~mol} / \mathrm{m}^{3}\right)$. These results suggest that as expected the use of therapeutic lenses induces a delay in the delivery of drug. This fact is in agreement with the findings in [16], where a simplest mathematical model has been used to simulate the two procedures of drug delivery.

The plots in Figure 12 indicate that when a therapeutic lens is used not only the release procedure is sustained as the drug concentration attains larger values.

\subsection{Treatment Simulations}

We concluded in Section 3.1 that, as expected, not only drug delivery is delayed when therapeutic lenses are used as drug concentration in the anterior chamber attains larger values. Therefore in what follows we simulate the evolution of drug concentration when therapeutic lenses are used for the delivery of antiglaucomatous medications. This means that we couple the dynamics of $\mathrm{AH}$ with glaucoma treatments with two distinct types of drugs: beta-blockers that inhibit the production rate of $\mathrm{AH}$ and prostaglandin derivatives that increase the $\mathrm{AH}$ outflow.

To simulate the kinetics of the drug in the TM we consider diffusion in porous media using the equation

$$
\epsilon \frac{\partial C_{T M}}{\partial t}+\nabla\left(\mathbf{u} C_{T M}\right)=\bar{D} \nabla^{2} C_{T M}, \text { in } \Omega_{2}, \Omega_{3}, t>0,
$$

where $C_{T M}$ represents the drug concentration in the trabecular mesh, $\bar{D}$ stands for the diffusion-dispersion coefficient $\left(\bar{D}=\epsilon D_{m} I+D_{d}, D_{d}=\alpha_{1}\|\mathbf{u}\|+\left(\alpha_{2}-\alpha_{1}\right) \frac{u u^{t}}{\|\mathbf{u}\|}\right)$ and $\epsilon$ is the porosity of the TM. In the last equation $\mathbf{u}$ represents the velocity of the AH in the TM, given by equation (2).

The values of the parameters used in the simulations are physically sound and are exhibited in Annex I. In this subsection we consider the whole problem (1-2),(4-6,11), defined in the geometry in Figure 4 where a therapeutical lens, $\Omega_{5}$, in contact with the cornea is considered. We recall that $\partial \Omega_{4,5}$ represents the boundary between the cornea and the therapeutic lens. The initial and boundary conditions for the concentration are

$$
\left\{\begin{array}{c}
C=C_{0}, \text { in } \Omega_{5}, t=0 \\
C^{b}=C_{0}^{b}, \text { in } \Omega_{5}, t=0 \\
C_{c}=0, \text { in } \Omega_{4}, t=0 \\
C_{T M}=0, \text { in } \Omega_{2}, \Omega_{3}, t=0 \\
C_{a}=0, \text { in } \Omega_{1}, t=0
\end{array}\right.
$$


and

$$
\left\{\begin{array}{c}
D \nabla C \cdot \eta=D_{c} \nabla C_{c} \cdot \eta, \text { in } \partial \Omega_{5,4}, t>0 \\
D_{c} \nabla C_{c} \cdot \eta=A_{c}\left(C_{c}-C a\right), \text { in } \partial \Omega_{1,4}, t>0 \\
D_{a} \nabla C_{a} \cdot \eta=A_{c}\left(C_{a}-C_{T M}\right), \text { in } \partial \Omega_{1,2}, t>0 \\
D \nabla C_{a} \cdot \eta=k\left(C_{T M}-C_{e x t}\right), \text { in } \partial^{o u t} \Omega_{2}, t>0
\end{array} .\right.
$$

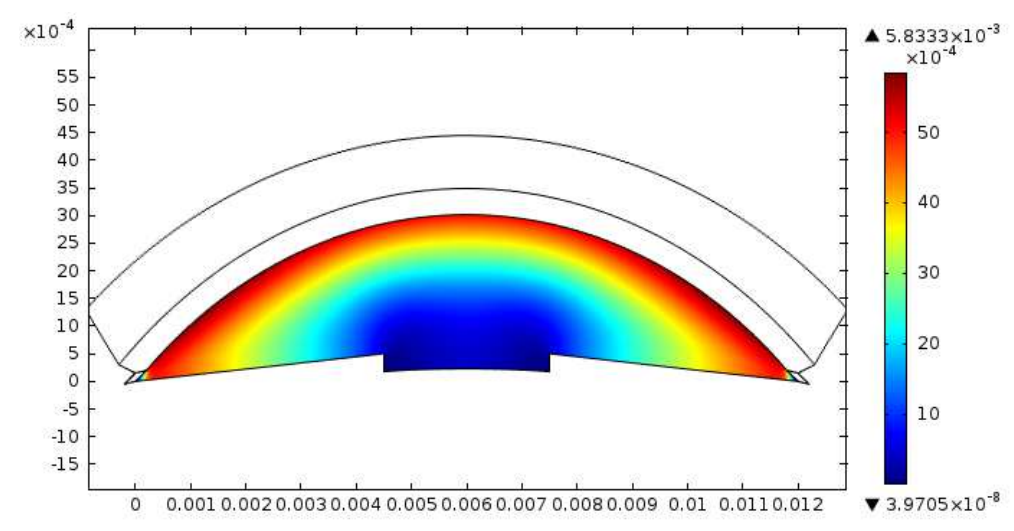

Figure 13: Drug concentration after $8 \mathrm{~h}$.

In Figure 13 we exhibit a 2D simulation of the drug concentration in the anterior chamber at $t=8 \mathrm{~h}$. The drug concentration in the lens and cornea is not exhibited. In this first simulation the production rate of $\mathrm{AH}$ has been kept constant as well as the porosity of the TM.

Our focus are two specific areas of the anterior chamber: the neighboring tissues of the ciliary body adjacent to $\partial \Omega_{4}$ and $\partial \Omega_{5}$, and the boundary tissues between the trabecular mesh and the anterior chamber, $\partial \Omega_{6}$ and $\partial \Omega_{7}$. In fact we assume that the total mass of drug that arrives at each one of these two regions represents a measure of the therapeutic effect of beta-blockers and prostaglandin derivatives respectively.

To simulate the decrease of $\mathrm{AH}$ rate under the effect of beta-blockers we define the total mass of drug in the neighborhoods of the ciliary body, as

$$
M_{I}(t)=\frac{1}{m\left(\partial \Omega_{4} \cup \partial \Omega_{5}\right)} \int_{0}^{t} \int_{\partial \Omega_{4} \cup \partial \Omega_{5}} C_{a}(\widehat{x}, s) d \widehat{x} d s,
$$

where $C_{a}$ stands for the concentration in the anterior chamber and $m\left(\partial \Omega_{4} \cup \partial \Omega_{5}\right)$ represents the length of $\partial \Omega_{4} \cup \partial \Omega_{5}$.

A plot of $M_{I}(t)$ is exhibited in Figure 14. In the first three hours the total mass of delivered drug represents around $50 \%$ of the total mass attained at $t=8 \mathrm{~h}$. We also observe that the plot of $M_{I}$ presents a steeper gradient in [0,3] than in $\left.] 3,8\right]$. We now use the plot of $M_{I}$ to establish the behaviour of AH rate under the effect of beta-blockers (Figure 15). As time evolves, $M_{I}(t)$ increases and the drug acts on the ciliary body decreasing the production 


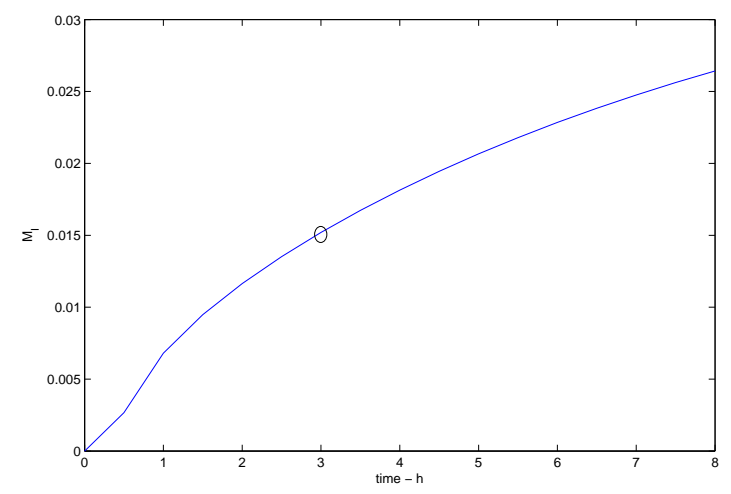

Figure 14: Behaviour of $M_{I}$ in the first $8 \mathrm{~h}$.

rate of $\mathrm{AH}$. In the plot of Figure 15 we assume that the initial rate $v_{0}$ lies within the range of normal values and that a decrease of $40 \%$ is observed after 8 hours. We also assume that, according to the behaviour of $M_{I}$, at $t=3 \mathrm{~h}$, the $\mathrm{AH}$ rate has decreases to around $20 \% v_{0}$, which corresponds to $50 \%$ of the final $\mathrm{AH}$ rate. To define the evolution of $v_{0}(t)$ in $[0,8]$ we complete the previous data with the values in Table III.

Table III - Interpolating points for $v_{0}(t)$.

\begin{tabular}{||c|c||}
\hline \hline time - t $(\mathrm{h})$ & Velocity \\
\hline 0 & $v_{0}$ \\
1 & $v_{0}-3 \% v_{0}$ \\
2 & $v_{0}-10 \% v_{0}$ \\
3 & $v_{0}-18 \% v_{0}$ \\
4 & $v_{0}-27 \% v_{0}$ \\
5 & $v_{0}-35 \% v_{0}$ \\
6 & $v_{0}-37.7 \% v_{0}$ \\
7 & $v_{0}-30.9 \% v_{0}$ \\
8 & $v_{0}-40 \% v_{0}$ \\
\hline \hline
\end{tabular}


Obviously that the ansatz used for defining $v_{0}(t)$ has an academic character. However at the best of our knowledge no experimental results exist relating the dependence of $v_{0}(t)$ on $M_{I}(t)$.

The plot of Figure 15 is obtained by quadratic interpolation of the values in Table III.

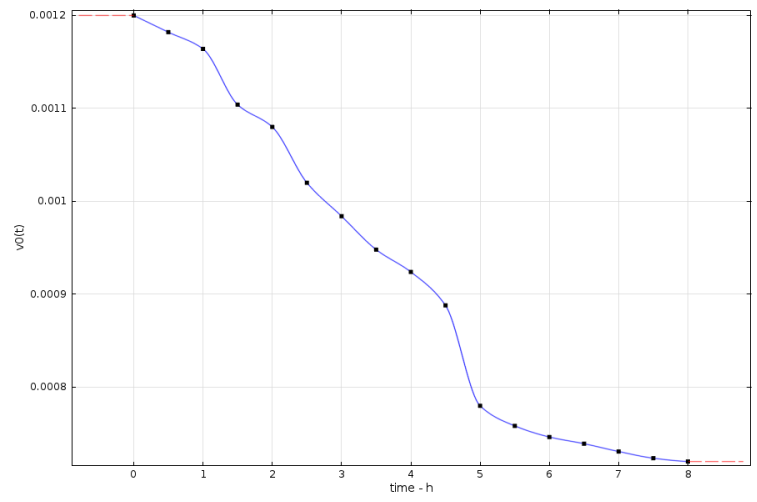

Figure 15: Behaviour of $v_{0}(t)$ in the first $8 \mathrm{~h}$.

To measure the IOP decrease after 8 hours we solve again the problem, but now with the boundary condition for the inlet velocity $v_{0}$ replaced by the data in Figure 15 . We simulate the effect of the beta-blocker treatment on IOP in a pathologic situation corresponding to a porosity $\epsilon=0.15$ which leads to a $p=3905$ (Table II). The effect of drug on the AH production rate is expected to decrease the IOP. We note that the porosity of TM is kept constant because beta-blockers just affect the production rate of $\mathrm{AH}$ and not its outflow. The behaviour of IOP is represented in Figure 16 (a).

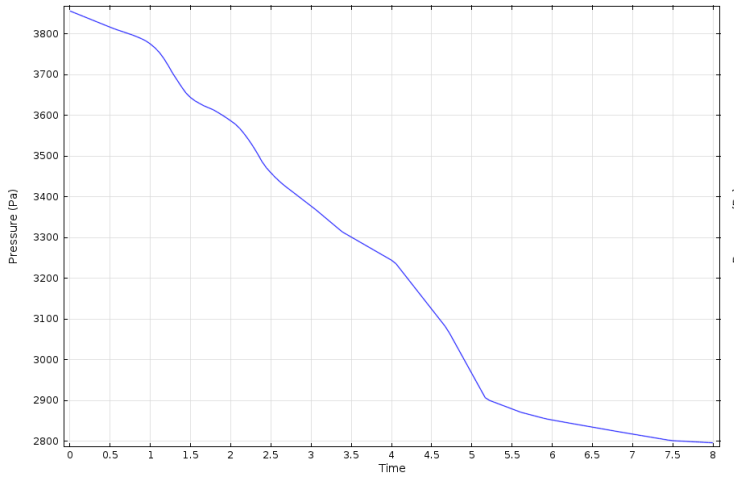

(a)

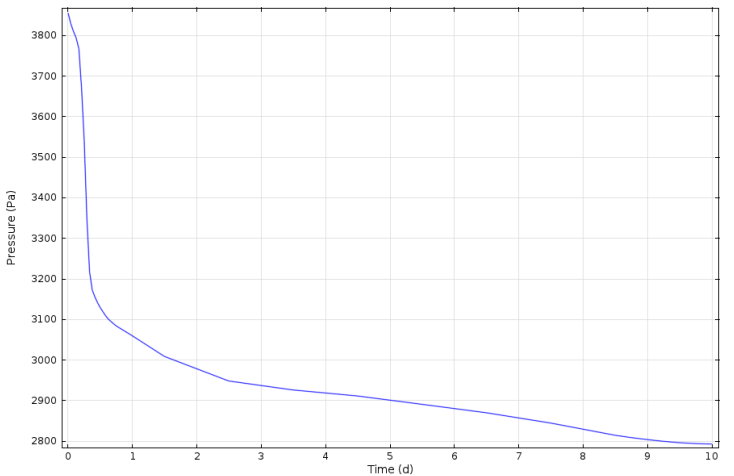

(b)

Figure 16: Behaviour of IOP under beta-blockers treatment assuming that $v_{0}(t)$ decreases by $40 \%$ at $t=8 h$ (a) and at $t=10$ days (b). 


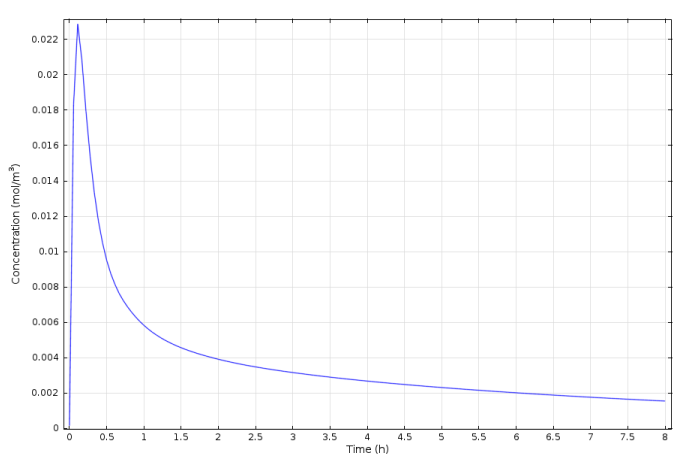

(a)

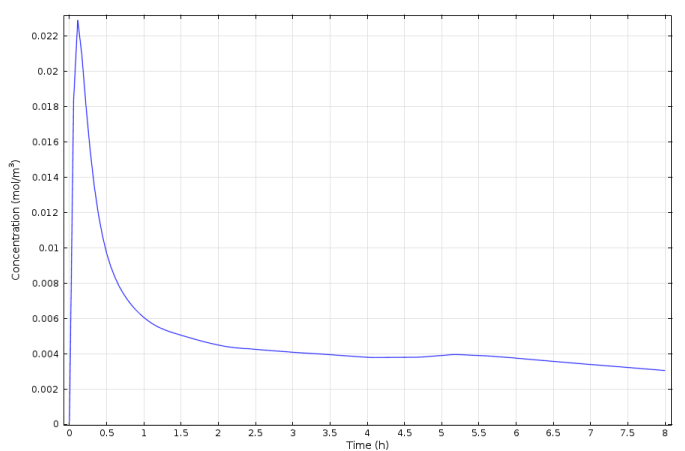

(b)

Figure 17: Behaviour of the mean concentration $C_{a}(t)$ in the first $8 \mathrm{~h}$ in $\partial \Omega_{4}$ : (a)- with $\mathbf{v}=v_{0}$ constant, (b) with $\mathbf{v}=v_{0}(t)$.

We observe that the plots of beta-blockers concentration, in the boundary of the anterior chamber, near the ciliary body, that is in $\partial \Omega_{4} \cup \partial \Omega_{5}$, when boundary condition $\mathbf{v}=v_{0}(t)$ or $\mathbf{v}=v_{0}$ are used present a different behaviour, as can be seen comparing Figure $17 a$ and $b$.

The behaviours in Figure 17 are physically sound: as $v_{0}(t) \leq v_{0}$, for each $t$, the advective effect of $\mathrm{AH}$, when beta-blockers are used, is less significant and the drug concentration is higher in $\partial \Omega_{4} \cup \partial \Omega_{5}$.

As we are simulating the delivery of beta-blockers through a therapeutic lens we should to plot the profile of IOP for a large period of time. Considering again an academic ansatz we assume that $v_{0}(t)$ decreases by $40 \%$ after 10 days the plot in Figure 16 (b) is obtained. The value of $v_{0}(t)$ at the interpolating points follows the previous procedure. We observe a steep gradient in the first two days followed by a soft decrease until day 10 .

Another family of drugs prescribed for reducing IOP are prostaglandin derivatives which increase the outflow of AH by increasing the permeability of the TM. To simulate a treatment with prostaglandins problem $(1-2),(4-6,11)$ is solved. To account for the therapeutic effect of the medication we consider that the porosity of the TM increases as the total mass that crosses $\partial \Omega_{1,2} U \partial \Omega_{1,3}$, that is the boundary between the anterior chamber and TM (Figure 8), increases. Let us represent that mass by

$$
M_{T M}(t)=-\int_{0}^{t} \int_{\partial \Omega_{1,2} \cup \partial \Omega_{1,3}} D_{a} \nabla C_{a}(\widehat{x}, s) d \widehat{x} d s .
$$

A plot of $M_{T M}$, where $C_{a}(\widehat{x}, s)$ is computed with a constant production rate $v_{0}$ and constant porosity $\epsilon$, is represented in Figure 18. We note that in the first 8 hours the total mass is increasing and the steady state is not already attained. For $t \in[0,3], M_{T M}(t)$ increases to $50 \%$ of the total mass at $t=8 \mathrm{~h}$. We consider a pathologic situation characterized by a very low porosity $\epsilon_{0}=0.15$ (Table II) and we assume that under the effect of the prostaglandin 


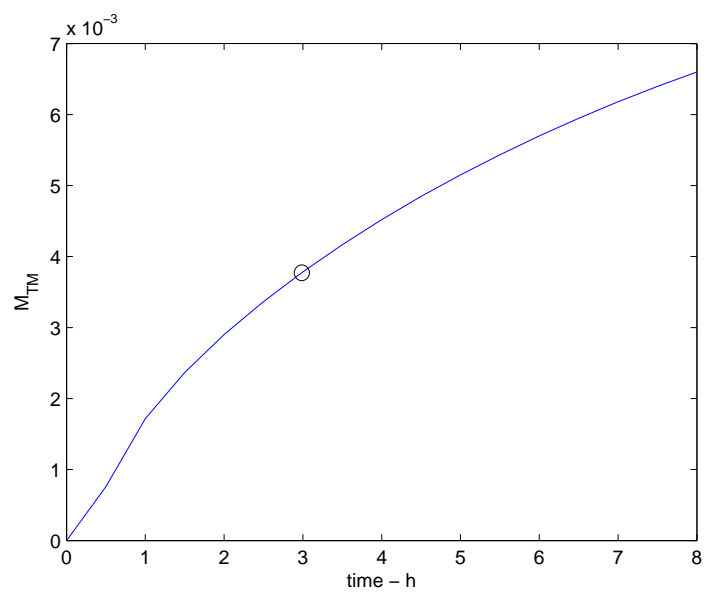

Figure 18: Behaviour of $M_{T M}$ in the first $8 \mathrm{~h}$.

treatment the porosity evolves following the same increasing trend as $M_{T M}(t)$. Consequently the evolution of $\epsilon$ is defined by an interpolation function built from the values in Table IV.

Table IV - Interpolating points for $\epsilon(t)$.

\begin{tabular}{||c|c||}
\hline \hline time - t $(\mathrm{h})$ & Porosity \\
\hline 0 & $\epsilon_{0}$ \\
2 & $\epsilon_{0}+12 \% \epsilon_{0}$ \\
3 & $\epsilon_{0}+20 \epsilon_{0}$ \\
6 & $\epsilon_{0}+30 \epsilon_{0}$ \\
8 & $\epsilon_{0}+1 / 3 \epsilon_{0}$ \\
\hline \hline
\end{tabular}

In Figure 19 we represent the evolution of the porosity in time. In a second computation the whole problem $(1)-(2),(4-6,11)$ is solved again, with a time dependent porosity function as represented in Figure 19.

As before we note that the ansatz underlying the evolution of the porosity, under a prostaglandin analog treatment, has an academic character.

In Figure 20 (a) we represent the evolution in time of the IOP in the anterior chamber as the prostaglandin analog is delivered though a therapeutic lens. We observe that the IOP decreases with time, attaining a nearly healthy value after 8 hours. The values in Table IV are theoretical assumptions with no evidence. If we assume that the increase around $30 \%$ is observed after 10 days, and we use $M_{T M}(t)$, as before, a different behaviour of the porosity of TM is obtained. This behaviour leads to the IOP profile exhibited in Figure 20 (b).

The behaviour of the pressure in the TM after $8 h$ of treatment is plotted in Figure 21. We observe that the pressure in the neighborhood of the interface boundary between TM and 


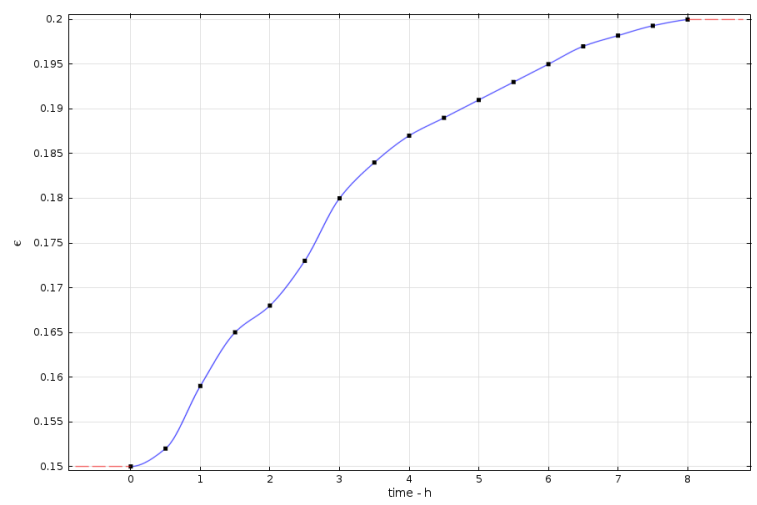

Figure 19: Behaviour of porosity in the first $8 \mathrm{~h}$.

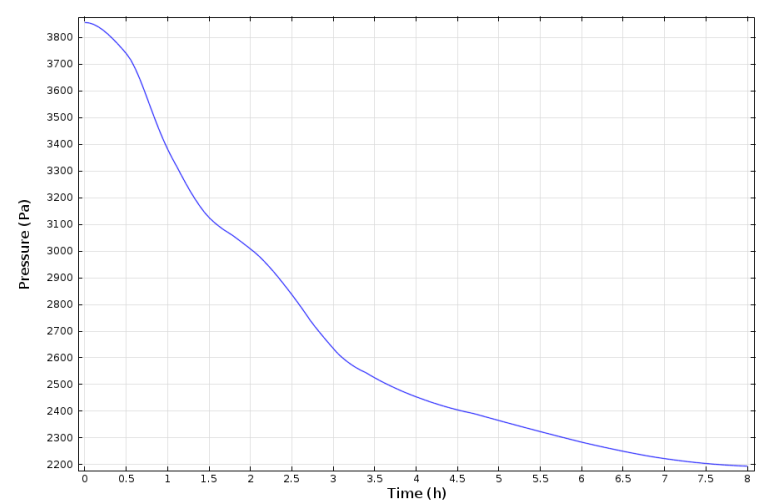

(a)

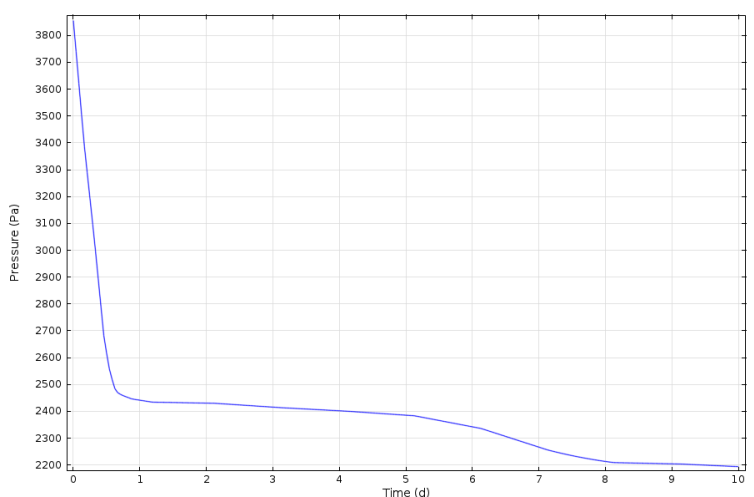

(b)

Figure 20: Behaviour of IOP under prostaglandin derivatives treatment assuming that $\epsilon(t)$ increases by around $30 \%$ at $t=8 h$ (a) and at $t=10$ days (b).

the anterior chamber is exactly $p=2200$, the same value obtained for the IOP in the anterior chamber (Figure 20).

\section{Conclusions}

In this paper a model to simulate the dynamics of aqueous humor in pathologic situations is presented. These pathologic situations are characterized by a high intraocular pressure. The two main factors related to the increase of IOP, which are production rate and drainage outflow, can be viewed as control parameters to decrease the pressure. In the first part of the paper (Section 2) the influence of these control parameters is explored. The dependence of the intraocular pressure on the drainage was simulated by considering different values for the permeability of the TM. The numerical results obtained suggest that abnormal values 


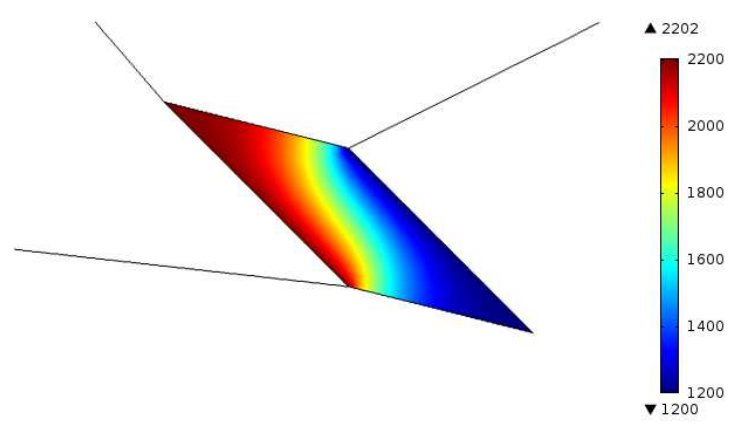

Figure 21: Behaviour of IOP in TM after $8 \mathrm{~h}$.

of intraocular pressure are visible only for very severe degrees of obstruction of TM (Table II). This finding is in agreement with clinical indications pointing out that low intraocular pressure is not enough to preclude the presence of glaucoma. The control of IOP by varying the production rate of aqueous humor provides an interesting information: its decrease induces a significant fall in the intraocular pressure of a pathologic eye whereas in healthy eyes a mild fall is observed (Table II). In the second part of the paper (Section 3) the behaviour of drug release through a therapeutic lens is compared to topic administration. The simulations presented show that the delivery through a lens presents a significant delay. The mathematical model used in the first part was then completed to include drug delivery through a therapeutic lens and the effects of treatments used to lower the intraocular pressure. Two different groups of drugs are considered: beta-blockers that decrease the production rate of aqueous humor and prostaglandin derivatives that increase the permeability and porosity of the TM. The plots representing the decrease of the production rate when beta-blockers are delivered (Figure 15) or the increase of porosity of the TM when prostaglandins are released(Figure 19) are in qualitative agreement with clinical results. However they are in a certain sense purely academic and based on medical acceptable ansatz. In fact to the best of our knowledge no experimental data exist in the literature to quantify such functional relations. The model shows in both cases a decrease of intraocular pressure after a certain period of time.

We believe that the models presented in this paper are helpful tools for clinicians because not only they give insight on the dynamics of $\mathrm{AH}$ under pathologic conditions but also on the mechanisms underlying the two main types of drugs used in the treatment of glaucoma. 


\section{A Annex}

\begin{tabular}{|c|c|}
\hline Symbol & Definition (unities) \\
\hline$v$ & velocity of the aqueous humor $(\mathrm{mm} / \mathrm{s})$ \\
\hline$p$ & pressure $(P a)$ \\
\hline$\epsilon$ & porosity \\
\hline$K$ & permeability $\left(m^{2}\right)$ \\
\hline$\rho$ & density $\left(\mathrm{kg} / \mathrm{m}^{3}\right)$ \\
\hline$\mu$ & $\operatorname{viscosity}(\mathrm{mm} / \mathrm{s})$ \\
\hline$D_{p}$ & pore size $(m m)$ \\
\hline$D_{l}$ & diffusion coefficient of the drug in the polymeric matrix $\left(\mathrm{m}^{2} / \mathrm{s}\right)$ \\
\hline$D_{c}$ & diffusion coefficient in the cornea $\left(\mathrm{m}^{2} / \mathrm{s}\right)$ \\
\hline$D_{a}$ & diffusion coefficient in the anterior chamber $\left(\mathrm{m}^{2} / \mathrm{s}\right)$ \\
\hline$C$ & drug concentration in the polymeric matrix $\left(\mathrm{mol} / \mathrm{m}^{3}\right)$ \\
\hline$C^{b}$ & drug concentration in the particles $\left(\mathrm{mol} / \mathrm{m}^{3}\right)$ \\
\hline$\lambda$ & transfer coefficient $\left(\mathrm{min}^{-1}\right)$ \\
\hline$C_{0}$ & initial concentration in the gel $\left(\mathrm{mol} / \mathrm{m}^{3}\right)$ \\
\hline$C_{0}^{b}$ & initial concentration in the particles $\left(\mathrm{mol} / \mathrm{m}^{3}\right)$ \\
\hline$C_{\text {ext }}$ & external concentration $\left(\mathrm{mol} / \mathrm{m}^{3}\right)$ \\
\hline$\alpha_{1}$ & transference coefficient $(\mathrm{cm} / \mathrm{min})$ \\
\hline$\gamma_{1}$ & rates distribution \\
\hline$C_{c}$ & drug concentration in the cornea $\left(\mathrm{mol} / \mathrm{m}^{3}\right)$ \\
\hline$C_{a}$ & drug concentration in the anterior chamber $\left(\mathrm{mol} / \mathrm{m}^{3}\right)$ \\
\hline$C_{T}$ & drug concentration in the tear film $\left(\mathrm{mol} / \mathrm{m}^{3}\right)$ \\
\hline$C_{T M}$ & drug concentration in the trabecular mesh $\left(\mathrm{mol} / \mathrm{m}^{3}\right)$ \\
\hline$K_{c}$ & metabolic consumption drug coefficient in the cornea \\
\hline$V_{a}$ & distribution volume of solute in the anterior chamber $(\mu l)$ \\
\hline$C l_{a}$ & clearance in the anterior chamber $(\mu l / \min )$ \\
\hline$A_{c}$ & surface area of the cornea $\left(\mathrm{cm}^{2}\right)$ \\
\hline$C_{f}$ & drug concentration in the tear film $\left(\mathrm{g} / \mathrm{cm}^{3}\right)$ \\
\hline$S$ & lacrimal secretion rate $(\mu l / \min )$ \\
\hline$k_{d}$ & drainage constant $\left(\mathrm{min}^{-1}\right)$ \\
\hline$V_{L}$ & normal lacrimal volume in tear film $(\mu l)$ \\
\hline$V_{i}$ & initial tear volume after an instillation of drug $(\mu l)$ \\
\hline
\end{tabular}

\section{Acknowledgements}

The authors thank João Pedro Alves, of Department of Physics and Mathematics, Coimbra Institute of Engineering, about the bibliography that he suggested about comsol.

This work was partially supported by the Centro de Matemática da Universidade de Coimbra (CMUC), funded by the European Regional Development Fund through the program COMPETE and by the Portuguese Government through the FCT - Fundação para a Ciência e Tecnologia under the projects PEst-C/MAT/UI0324/2011, SFR/BD/33703/2009, and by the project UTAustin/MAT/0066/2008. 


\section{References}

[1] Tripathi, R. C., and Tripathi, B. J. (1984): Anatomy of the human eye, orbit, and adnexa, in The Eye, Vol 1a, Vegetative physiology and biochemistry, edited by Hugh Davson, Academic Press.

[2] Manik Goel, Renata G Picciani, Richard K Lee, and Sanjoy K Bhattacharya, Aqueous Humor Dynamics: A Review Open Ophthalmol J. 2010, 4, 52-59.

[3] Rittenhouse K.D., Peiffer R.L. Jr., Pollack G.M., Evaluation of microdialysis sampling of aquaous humor for in vivi models of ocular absorption and disposition, PubMed - indexed for Medline, PMID: 9547698.

[4] Gulsen, D.; Chauhan, A., Dispersion of microemulsion drops in HEMA hydrogel: a potential ophthalmic drug delivery vehicle, International Journal of Pharmaceutics, vol.292, pp. 95-117, 2005 .

[5] Jennifer H. Siggers and C. Ross Ethier., Fluid Mechanics of the Eye, Annu. Rev. Fluid Mech, 44, 347-372, 2012.

[6] Jyoti Kathawate., Numerical solution of flow resistance in outflow pathway and intravitreal drug delivery in vitrectomised eyes, Master's thesis, Dept. Mech. Eng., Louisiana State University, December 2006.

[7] Fitt D., G. Gonzalez G., Fluid Mechanics of the Human Eye: Aqueous Humour Flow in the Anterior Chamber, Bull. Math. Biol., 68:53-71, 2006.

[8] T. Crowder, Vince Ervin., Numerical Simulations of Fluid Pressure in the Human Eye, to appear in Applied Mathematics and Computation.

[9] S. Kumar., Numerical solution of ocular fluid dynamics, Master's thesis, Dept. Mech. Eng., Louisiana State University, December 2003.

[10] Alan L. Robin, Efficacy and Safety are factor that must be considered when patients taking a prostaglandin require additional IOP reduction, Review of Ophtalmology, 2008.

[11] Ammar D.A., Malik Y. Kahook, Effects of benzalkonium chloride - or polyqaud - preserved fixed combination glaucoma medications on human trabecular meshwork cells, Mol Vis. 2011;17:1806-13. Epub 2011 Jul 2.

[12] Carol B. Toris, B' Ann T. Gabelt, Paul L. Kaufman, Update on the Mechanism of Action of Topical Prostaglandins for Intraocular Pressure Reduction, MD2 Surv Ophthalmol. 2008 November; 53(SUPPL1): S107-S120.

[13] Rittenhouse K.D., Pollack G.M., Pharmacodynamics of beta-blocker modulation of aqueous humor production, PubMed - indexed for Medline, PMID: 10865991.

[14] Ciolino, J. B.; Hoare, T. R.; Iwata, N. G.; Behlau, I.; Dohlman, C. H.; Langer, R.; Kohane, D.S. (2009): A Drug-Eluting Contact Lens, Investigative Ophthalmology \& Visual Science, vol.50, 7. 
[15] Ferreira, J. A.; Oliveira, P.; Silva, P. M.; Carreira, A.; Gil, H.; Murta, J. N. (2010): Sustained drug release from contact lens, Computer Modeling in Engineering and Science, vol.60, pp. 152-179.

[16] Ferreira, J. A.; Oliveira, P.; Silva, P. M.; Murta, J. N. (2011): Drug delivery: from a ophthalmic lens to the anterior chamber, Computer Modeling in Engineering and Science.

[17] Gulsen, D.; Chauhan, A. (2004): Ophthalmic drug delivery from contact lenses, Investigative Ophthalmology and Visual Science, vol.45, pp. 2342-2347.

[18] Silva, P.M. (2010): Controlled Drug Delivery: Analytical and Numerical Study, Phd-Thesis, University of Coimbra, Portugal.

[19] Kapnisis K, Doormaal MV, C. Ross Ethier., Modeling aqueous humor collection from the human eye, J Biomech., 42, 2454-2457, 2009.

[20] Silver DM, Quigley HA., Aqueous flow through the iris-lens channel: estimates of differential pressure between the anterior and posterior chambers, J Glaucoma, 13, 100-107, 2004.

[21] Peng, C. C., Bruke, M. T., Carbia B.E., Plummer C., Chauhan A. Extended drug delivery by contact lenses for glaucoma therapy, J. Control Release, 162, 152-158, 2012.

[22] Zhang W, Prausnitz MR, Edwards A. Model for transient drug diffusion across cornea, J Control Release 2004; 99: 241-258.

[23] Avtar R, Tandon D., Modeling the drug transport in the anterior segment of the eye, Eur J Pharm Sci 2008; 35:175-182. 